\title{
Temporal Data Mining for Neuroscience
}

\author{
Wu-chun Feng, Yong Cao, Debprakash Patnaik, and Naren Ramakrishnan \\ Department of Computer Science \\ Virginia Tech
}

Today, multi-electrode arrays (MEAs) capture neuronal spike streams in real time, thus providing dynamic perspectives into brain function. Mining such spike streams from these MEAs is critical towards understanding the firing patterns of neurons and gaining insight into the underlying cellular activity. However, the acquisition rate of neuronal data places a tremendous computational burden on the subsequent temporal data mining of these spike streams. Thus, computational neuroscience seeks innovative approaches towards tackling this problem and eventually solving it efficiently and in real time.

In this chapter, we present a solution that uses graphics processing units (GPUs) to mine spike train datasets. Specifically, our solution delivers a novel mapping of a "finite state machine for data mining" onto the GPU while simultaneously addressing a wide range of neuronal input characteristics. This solution ultimately transforms the task of temporal data mining of spike trains from a batch-oriented process towards a real-time one.

\subsection{Introduction}

Brain-computer interfaces have made massive strides in recent years [1]. Scientists are now able to analyze neuronal activity in living organisms, understand the intent implicit in these signals and, more importantly, use this information as control directives to operate external devices. Technologies for modeling and recording neuronal activity include functional magnetic resonance imaging (fMRI), electroencephalography (EEG), and multi-electrode arrays (MEAs).

Here we focus on event streams gathered through MEA chips for studying neuronal function. As shown in Figure 1.1, a MEA records spiking action potentials from an ensemble of neurons which, after various pre-processing steps, yields a spike train dataset that provides a real-time dynamic perspective into brain function. Key problems of interest include identifying sequences of firing neurons, determining their characteristic delays, and reconstructing 


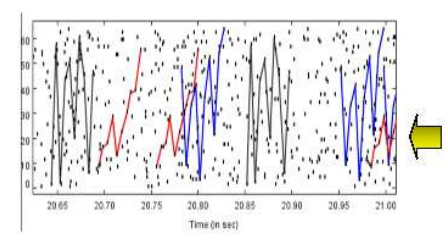

Frequent Episodes
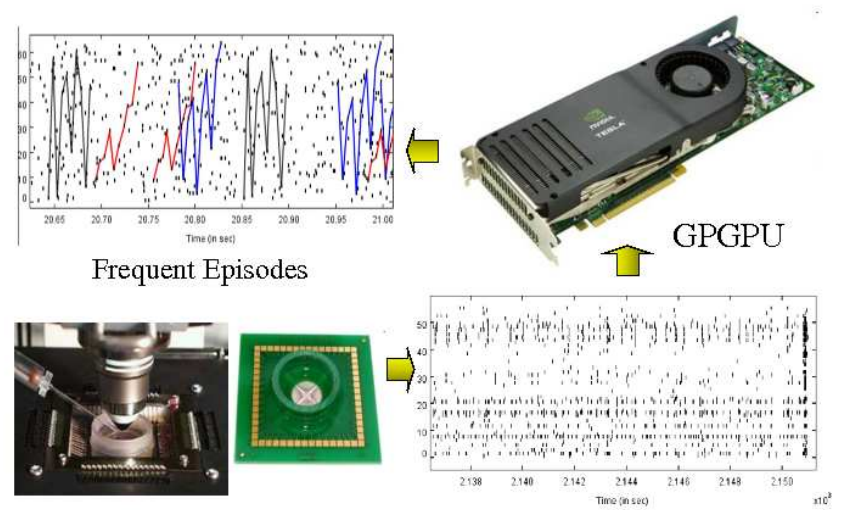

Micro-electrode Array

Action Potentials from real neuron cells

Fig. 1.1. Spike trains recorded from a multi-electrode array (MEA) are mined by a GPU to yield frequent episodes, which can be summarized to reconstruct the underlying neuronal circuitry.

the functional connectivity of neuronal circuits. Addressing these problems can provide critical insights into the cellular activity recorded in the neuronal tissue.

In only a few minutes of cortical recording, a 64-channel MEA can easily capture millions of neuronal spikes. In practice, such MEA experiments run for days or even months [8] and can result in trillions to quadrillions of neuronal spikes. From these neuronal spike streams, we seek to identify (or mine for) frequent episodes of repetitive patterns that are associated with higher-order brain function. The mining algorithms of these patterns are usually based on finite state machines $[3,5]$ and can handle temporal constraints [6]. The temporal constraints add significant complexity to the state machine algorithms as they must now keep track of what part of an episode has been seen, which event is expected next, and when episodes interleave. Then, they must make a decision of which events to be used in the formation of an episode.

\subsection{Core Methodology}

We model a spike train dataset as an event stream, where each symbol/event type corresponds to a specific neuron (or clump of neurons). In addition, the dataset encodes the occurrence times of these events.

Temporal data mining of event streams aims to discover interesting patterns that occur frequently in the event stream, subject to certain timing constraints. More formally, each pattern, i.e., episode, is an ordered tuple of 
event types with temporal constraints. For example, the episode shown below describes a pattern where event $B$ must occur at least $t_{\text {low }}^{1}$ after event $A$ and at most $t_{\text {high }}^{1}$ after event $A$ and event $C$ must occur at least $t_{\text {low }}^{2}$ after event $B$ and at most $t_{\text {high }}^{2}$ after event $B$.

$$
\left(A \stackrel{\left(t_{\text {low }}^{1}, t_{\text {high }}^{1}\right]}{\longrightarrow} B \stackrel{\left(t_{\text {low }}^{2}, t_{\text {high }}^{2}\right]}{\longrightarrow} C\right)
$$

The above is a size- 3 episode since it contains 3 events in the episode.

Episodes are mined by a generate-and-test approach, i.e., we generate numerous candidate episodes which are counted to ascertain their frequencies. This process happens levelwise: search for 1-node episodes (single symbols) first, followed by 2-node episodes, and so on. At each level, size- $N$ candidates are generated from size- $(N-1)$ frequent episodes, and their frequencies (i.e., counts) are determined by making a pass over the event sequence. Only those candidate episodes whose count is greater than a user-defined threshold are retained. The most computationally expensive step in each level is the counting of all candidate episodes for that level. At the initial levels, there are a large number of candidate episodes to be counted in parallel; the later levels only have increasingly fewer candidate episodes.

The frequency of an episode is a measure that is open to multiple interpretations. Note that episodes can have "junk" symbols interspersed, e.g., an occurrence of event A followed by B followed by C might have symbol D interspersed whereas a different occurrence of the same episode might have symbol F interspersed. Example ways to define frequency include counting all occurrences, counting only non-overlapped occurrences, and so on. We utilize the non-overlapped count, which is defined as the maximum number of nonoverlapped instances of the given episode. This measure has the advantageous property of anti-monotonicity, i.e., the frequency of a sub-episode cannot be less than the frequency of the given episode. Anti-monotonicty allows us to use level-wise pruning algorithms in our search for frequent episodes.

Our approach is based on a state machine algorithm with inter-event constraints [6]. Algorithm 1 shows our serial counting procedure for a single episode $\alpha$. The algorithm maintains a data structure $s$, which is a list of lists. Each list $s[k]$ in $s$ corresponds to an event type $E_{(k)} \in \alpha$ and stores the times of occurrences of those events with event type $E_{(k)}$ that satisfy the inter-event constraint $\left(t_{\text {low }}^{(k-1)}, t_{h i g h}^{(k-1)}\right]$ with at least one entry $t_{j} \in s[k-1]$. This requirement is relaxed for $s[0]$, thus every time an event $E_{(0)}$ is seen in the data, its occurrence time is pushed into $s[0]$.

When an event of type $E_{(k)}, 2 \leq k \leq N$ at time $t$ is seen, we look for an entry $t_{j} \in s[k-1]$ such that $t-t_{j} \in\left(t_{\text {low }}^{(k-1)}, t_{\text {high }}^{(k-1)}\right]$. Therefore, if we are able to add the event to the list $s[k]$, it implies that there exists at least one previous event with event type $E_{(k-1)}$ in the data stream for the current event that satisfies the inter-event constraint between $E_{(k-1)}$ and $E_{(k)}$. Applying this argument recursively, if we can add an event with event type $E_{(|\alpha|)}$ to its 


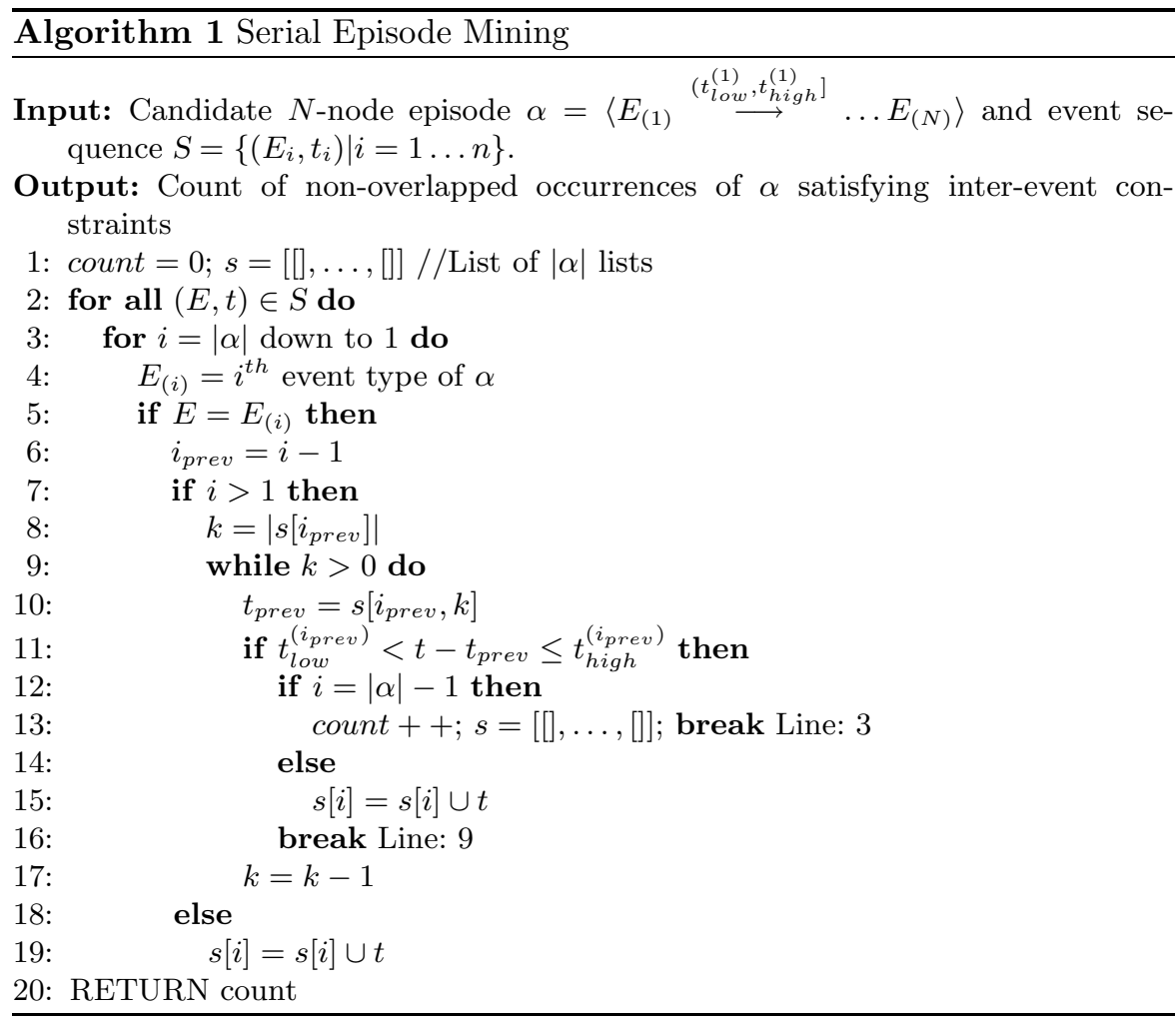

corresponding list in $s$, then there exists a sequence of events corresponding to each event type in $\alpha$ satisfying the respective inter-event constraints. Such an event marks the end of an occurrence, after which the count for $\alpha$ is incremented and the data structure $s$ is reinitialized. Figure 1.2 illustrates the data structure $s$ for counting $A \stackrel{(5,10]}{\rightarrow} B \stackrel{(10,15]}{\rightarrow} C$.

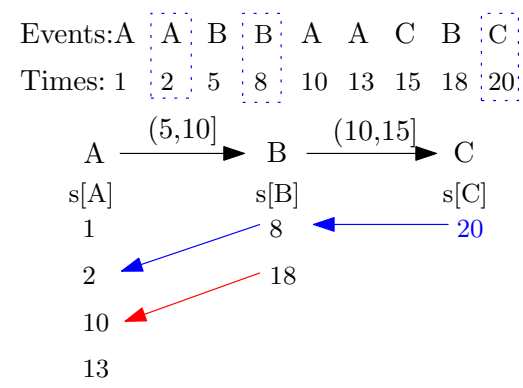

Fig. 1.2. Illustration of the data structure $s$ for counting $A \stackrel{(5,10]}{\longrightarrow} B \stackrel{(10,15]}{\rightarrow} C$ 


\subsection{GPU Parallelization: Algorithms and Implementations}

To parallelize the above sequential counting approach on a GPU, we segment the overall computation into independent units that can be mapped onto GPU cores and executed in parallel to fully utilize GPU resources. Different computation-to-core mapping schemes can result in different levels of parallelism, which are suitable for different inputs for the episode counting algorithm. Next, we present two computation-to-core mapping strategies, which are suitable for different scenarios with different sizes of input episodes. The first strategy, one thread per occurrence of an episode, is used for mining a very few number of episodes. The second strategy is used for counting a large number of episodes.

\subsubsection{Strategy 1: One Thread per Occurrence}

\section{Problem Context}

This mapping strategy handles the case when we have a few input episodes to count. In the limit, mining one episode with one thread severely under-utilizes the GPU. Thus, we seek an approach to increase the level of parallelism. The original sequential version of the mining algorithm uses a state-machine approach with a substantial amount of data dependencies. Hence, it is difficult to increase the degree of parallelism by optimizing this algorithm directly. Instead, we transform the algorithm by discarding the state-machine approach and decomposing the problem into two sub-problems that can be easily parallelized using known computing primitives. The mining of an episode entails counting the frequency of non-overlapped neuronal patterns of event symbols. It represents the size of the largest set of non-overlapped occurrences. Based on this definition, we design a more data-parallel solution.

\section{Basic Idea}

In our new approach, we find a superset of the non-overlapped occurrences that could potentially be overlapping. Each occurrence of an episode has a start time and an end time. If each episode occurrence in this superset is viewed as a task/job with fixed start and end times, then the problem of finding the largest set of non-overlapped occurrences transforms itself into a job scheduling problem, where the goal is to maximize number of jobs or tasks while avoiding conflicts. A greedy $O(n)$ algorithm solves this problem optimally where $n$ is the number of jobs. The original problem now decomposes into the following two sub-problems:

1. Find a superset of non-overlapped occurrences of an episode.

2. Find the size of the largest set of non-overlapped occurrences from the above set of occurrences. 
The first sub-problem can be solved with a high degree of parallelism as will be shown below. We design a solution where one GPU thread can mine one occurrence of an episode. To pre-process the data, however, we perform an important compaction step between the searches of the next query event in the episode. This entailed us to investigate both a lock-based compaction method using atomic operations in CUDA as well as a lock-free approach with the CUDPP library. However, the performance of both compaction methods was unsatisfactory. Thus, in order to further improve performance, we adopted a counter-intuitive approach that divided the counting process into three parts. First, each thread looks up the event sequence for suitable next events but instead of recording the events found, it merely counts and writes the count to global memory. Second, an exclusive scan is performed on the recorded counts. This gives the offset into the global memory where each thread can write its 'next events' list. The actual writing is done as the third step. Although each thread looks up the event sequence twice (first to count and second to write), we show that we nevertheless achieve better performance.

The second sub-problem is the same as the task or interval scheduling problem where tasks have fixed times. A fast greedy algorithm is well known to solve the problem optimally.

\section{Algorithmic Improvements}

We first pre-process the entire event stream noting the positions of events of each event type. Then for a given episode, beginning with the list of occurrences of the start event-type in the episode, we find occurrences satisfying the temporal constraints in parallel. Finally, we collect and remove overlapped occurrences in one pass. The greedy algorithm for removing overlaps requires the occurrences to be sorted by end time, and the algorithm proceeds as shown in Algorithm 2. Here, for every set of consecutive occurrences, if the start time is after the end time of the last selected occurrence, then we select this occurrence, otherwise we skip it and go to the next occurrence.

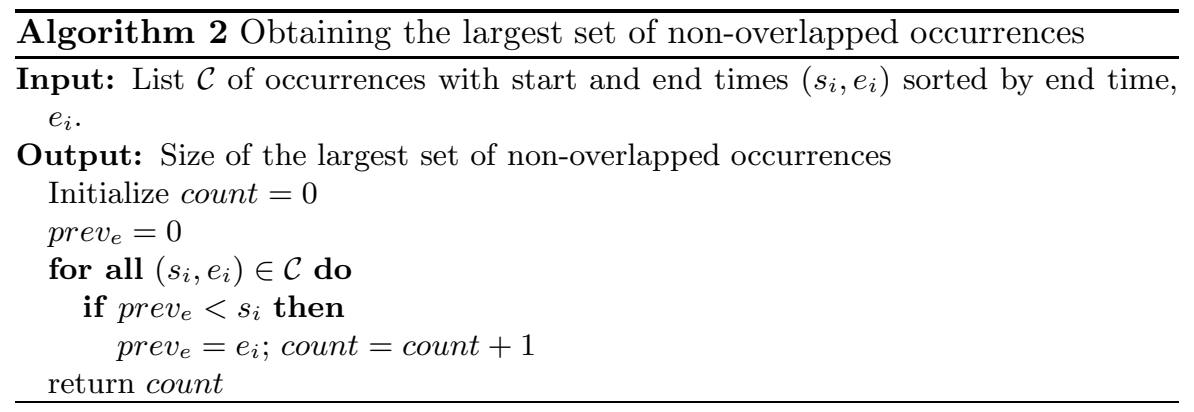

Next we explore different approaches of solving the first sub-problem, as presented earlier. The aim here is to find a super-set of non-overlapped oc- 
currences in parallel. The basic idea is to start with all events of the first event-type in parallel for a given episode and find occurrences of the episode starting at each of these events. There can be several different ways in which this can be done. We shall present two approaches that gave us the most performance improvement. We shall use the episode $A \stackrel{(5-10]}{\longrightarrow} B \stackrel{(5-10]}{\longrightarrow} C$ as our running example and explain each of the counting strategies using this example. This example episode specifies event occurrences where an event A is to be followed by an event $\mathrm{B}$ within $5-10 \mathrm{~ms}$ and event $\mathrm{B}$ is to be followed by an event $\mathrm{C}$ within $5-10 \mathrm{~ms}$ delay. Note again that the delays have both a lower and an upper bound.

\section{Parallel local tracking}

In the pre-processing step, we have noted the locations of each of the eventtypes in the data. In the counting step, we launch as many threads as there are events in the event stream of the start event-type (of the episode). In our running example these are all events of type $A$. Each thread searches the event stream starting at one of these events of type $A$ and looks for an event of type $B$ that satisfies the inter-event time constraint $(5-10]$ i.e., $5<t_{B_{j}}-t_{A_{i}} \leq 10$ where $i, j$ are the indices of the events of type $A$ and $B$. One thread can find multiple $B$ 's for the same $A$. These are recorded in a preallocated array assigned to each thread. Once all the events of type $B$ (with an $A$ before them) have been collected by the threads (in parallel), we need to compact these newfound events into a contiguous array/list. This is necessary as in the next kernel launch we will find all the events of type $C$ that satisfy the inter-event constraints with this set of B's. This is illustrated in Figure 1.3.

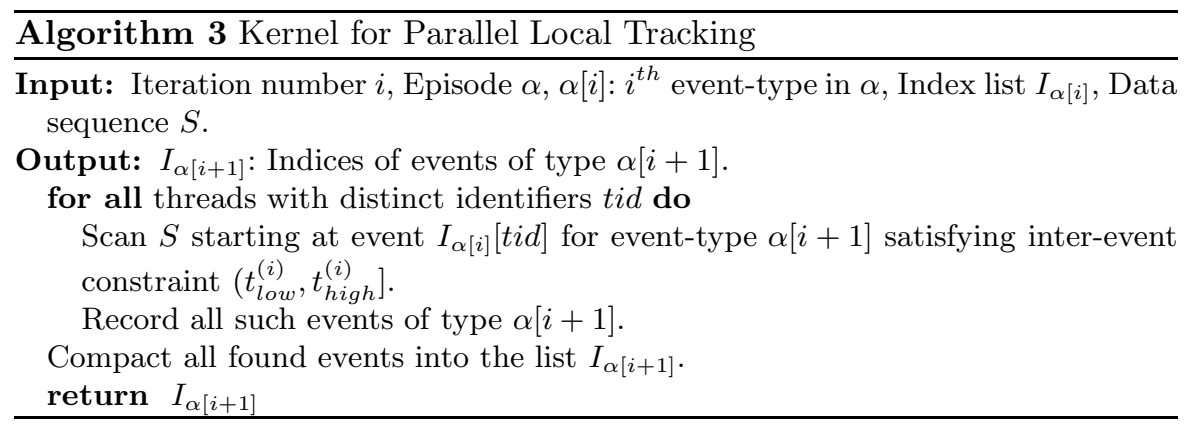

Algorithm 3 presents the work done in each kernel launch. In order to obtain the complete set of occurrences of an episode, we need to launch the kernel $N-1$ times where $N$ is the size of an episode. The list of qualifying events found in the $i^{\text {th }}$ iteration is passed as input to the next iteration. Some amount of bookkeeping is also done to keep track of the start and end times of an occurrence. After this phase of parallel local tracking is completed, the 

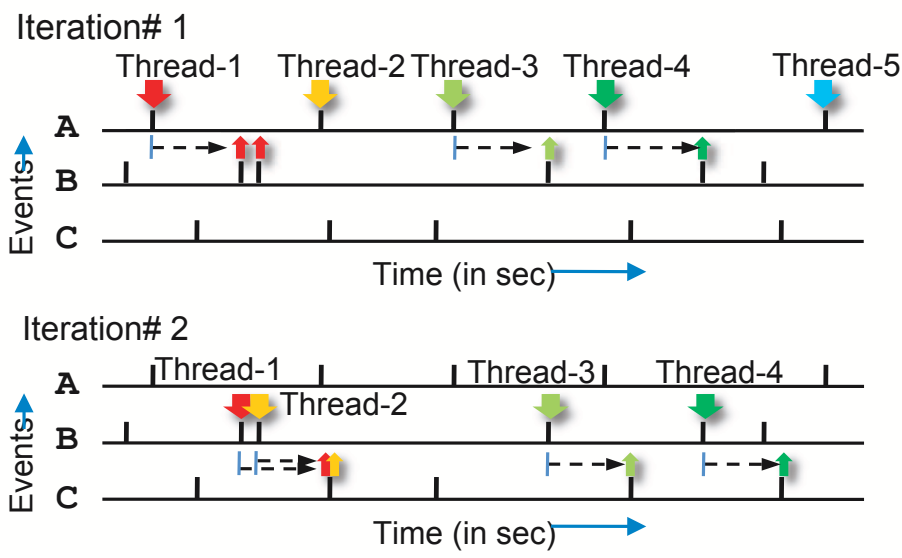

Fig. 1.3. Illustration of the parallel local tracking algorithm (i.e., Algorithm 3), showing 2 iterations for the episode $A \rightarrow B \rightarrow C$ with implicit inter-event constraints. Note that each thread can find multiple next-events. Further, a thread stops scanning the event sequence when event-times go past the upper bound of the inter-event constraint.

non-overlapped count is obtained using Algorithm 2. The compaction step in Algorithm 3 presents a challenge as it requires concurrent updates into a global array.

\section{Implementation Notes}

\section{Lock-based compaction}

NVIDIA graphics cards with CUDA compute capability 1.3 support atomic operations on shared and global memory. Here we use atomic operations to perform compaction of the output array into the global memory. After the counting step, each thread has a list of next-events. Subsequently, each thread adds the size of its next-events list to the block-level counter using an atomic add operation and, in return, obtains a local offset (which is the previous value of the block-level counter). After all threads in a block have updated the blocklevel counter, one thread from a block updates the global counter by adding the value of the block-level counter to it and, as before, obtains the offset into global memory. Now all threads in the block can collaboratively write into the correct position in the global memory (resulting in overall compaction). A schematic for this operation is shown for 2-blocks in Figure 1.4. In the results section, we refer to this method as Atomic Compact.

Since there is no guarantee for the order of atomic operations, this procedure requires sorting. The complete occurrences need to be sorted by end time for Algorithm 2 to produce the correct result. 


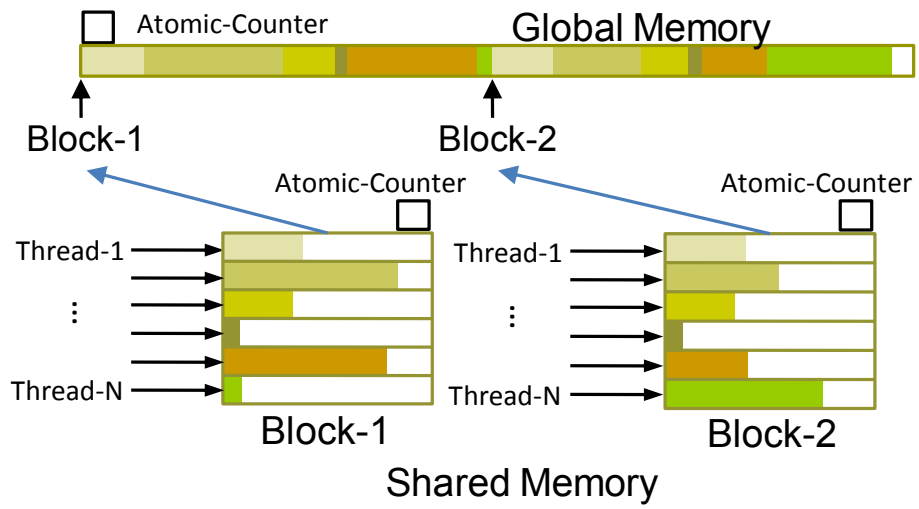

Fig. 1.4. Illustration of output compaction using AtomicAdd operations. Note that we use atomic operations at both block and global level. These operations return the correct offset into global memory for each thread to write its next-event list into.

\section{Lock-free compaction}

Prefix scan is known to be a general-purpose, data-parallel primitive that is a useful building block for algorithms in a broad range of applications. Given a vector of data elements $\left[x_{0}, x_{1}, x_{2}, \ldots\right]$, an associative binary function $\oplus$ and an identity element $i$, exclusive prefix scan returns $\left[i, x_{0}, x_{0} \oplus x_{1}, x_{0} \oplus\right.$ $\left.x_{1} \oplus x_{2}, \ldots\right]$. The first parallel prefix-scan algorithm was proposed in 1962 [4]. With increasing interest in general-purpose computing on the GPU (GPGPU), several implementations of scan have been proposed for GPU, the most recent ones being [2] and [7]. The latter implementation is available as the CUDPP: CUDA Data Parallel Primitives Library and forms part of the CUDA SDK distribution.

Our lock-free compaction is based on prefix sum, and we reuse the implementation from CUDPP library. Since the scan-based operation guarantees ordering, we modify our counting procedure to count occurrences backwards starting from the last event. This results in the final set of occurrences to be automatically ordered by end time and therefore completely eliminates the need for sorting (as required by the approach based on atomic operations).

The CUDPP library provides a compact function which takes an array $d_{i n}$, an array of $1 / 0$ flags, and returns a compacted array $d_{\text {out }}$ of corresponding only the "valid" values from $d_{i n}$ (it internally uses cudppScan). In order to use this, our counting kernel is now split into three kernel calls. Each thread is allocated a fixed portion of a larger array in global memory for its next-events list. In the first kernel, each thread finds its events and fills up its next-events list in global memory. The cudppCompact function, implemented as two GPU kernel calls, compacts the large array to obtain the global list of next-events. A difficulty of this approach is that the array on which cudppCompact operates 


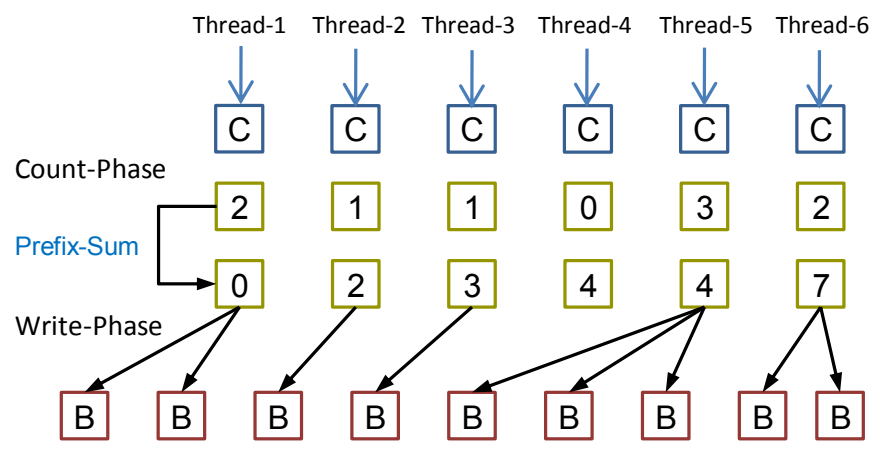

Fig. 1.5. Illustration of output compaction using the scan primitive. Each iteration is broken into 3 kernel calls: Counting the number of next events, Using scan to compute offset into global memory, finally launching count-procedure again but this time allowing write operations to the global memory

is very large resulting in a scattered memory access pattern. We refer to this method as CudppCompact.

In order to further improve performance, we adopt a counterintuitive approach. We again divide the counting process into three parts. First, each thread looks up the event sequence for suitable next-events but instead of recording the events found, it merely counts and writes the count to global memory. Then an exclusive scan is performed on the recorded counts. This gives the offset into the global memory where each thread can write its nextevents list. The actual writing is done as the third step. Although each thread looks up the event sequence twice (first to count, and second to write), we show that we nevertheless achieve better performance. This entire procedure is illustrated in Figure 1.5. We refer to this method of compaction as CountScanWrite in the ensuing results section.

Note that prefix scan is essentially a sequential operator applied from left to right to an array. Hence, the memory writes operations into memory locations generated by prefix scan preserve order. The sorting step (i.e., sorting occurrences by end time) required in the lock-based compaction can be completely avoided by counting occurrences backwards, starting from the last event-type in the episode.

\subsubsection{Strategy 2: A Two-Pass Elimination Approach}

\section{Problem Context}

When mining a large number of input episodes, we can simply assign one GPU thread for each episode. Since there are enough episodes, the GPU computing resource will be fully utilized. The state-machine based algorithm is very complex and requires of a large amount of shared memory and large 
number of GPU registers for each GPU thread. For example, if the length of the query is 5 , each thread requires 220 bytes of shared memory and 97 bytes of register file. It means that only 32 threads can be allocated on a GPU multiprocessor, which has $16 \mathrm{~K}$ bytes of shared memory and register file. As each thread requires more resources, fewer threads can run on GPU at the same time, resulting in longer execution time for each thread.

\section{Basic Idea}

To address this problem, we sought to reduce the complexity of the algorithm without losing correctness. Our idea was to use a simpler algorithm that we call PreElim to eliminate most of non-supported episodes, and only use the more complex algorithm to determine if the rest of the episode is supported or not. To introduce the algorithm PreElim, we consider the solution to a slightly relaxed problem, which plays an important role in our two-pass elimination approach. In this approach, algorithm PreElim is simpler and runs faster than the more complex algorithm, because it reduces the time complexity of the inter-event constraint. As a result, when the number of episodes is very large and the number of episodes culled in the first pass is also large, the performance of our two-pass elimination algorithm is significantly better than the more complex, original algorithm.

\section{Algorithmic improvements}

Less-Constrained Mining: Algorithm PreElim.

Let us consider a constrained version of Problem 1. Instead of enforcing both lower-limits and upper-limits on inter-event constraints, we design a counting solution that enforces only upper limits.

Let $\alpha^{\prime}$ be an episode with the same event types as in $\alpha$, where $\alpha$ uses the original episode definition from Problem 1. The lower bounds on the interevent constraints in $\alpha$ are relaxed for $\alpha^{\prime}$ as shown below.

$$
\alpha^{\prime}=\left\langle E_{(1)}^{\stackrel{\left(0, t_{\text {high }}^{(1)}\right]}{\longrightarrow}} E_{(2)} \ldots \stackrel{\left(0, t_{h i g h}^{(N-1)}\right]}{\longrightarrow} E_{(N)}\right\rangle
$$

Observation 1.3.1 In Algorithm 1, if lower-bounds of inter-event constraints in episode $\alpha$ are relaxed as $\alpha^{\prime}$, the list size of $s[k], 1 \leq k \leq N$ can be reduced to 1 .

Proof. In Algorithm 1, when an event of type $E_{(k)}$ is seen at time $t$ while going down the event sequence, $s\left[E_{(k-1)}\right]$ is looked up for at least one $t_{i}^{k-1}$, such that $t-t_{i}^{k-1} \in\left(0, t_{h i g h}^{(k-1)}\right]$. Note that $t_{i}^{k-1}$ represents the $i^{t h}$ entry of $s\left[E_{(k-1)}\right]$ corresponding the $(k-1)^{t h}$ event-type in $\alpha$. 


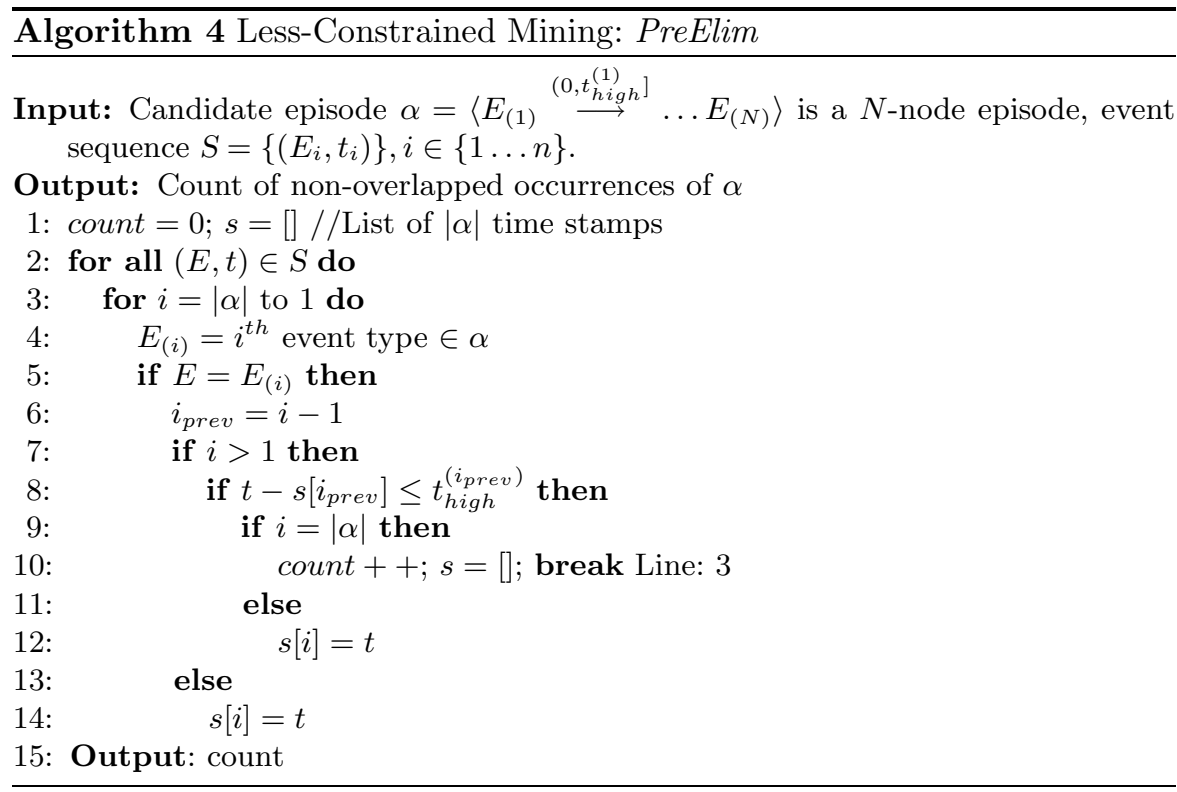

Let $s\left[E_{(k-1)}\right]=\left\{t_{1}^{k-1} \ldots t_{m}^{k-1}\right\}$ and $t_{i}^{k-1}$ be the first entry which satisfies the inter-event constraint $\left(0, t_{h i g h}^{(k-1)}\right]$, i.e.,

$$
0<t-t_{i}^{k-1} \leq t_{h i g h}^{(k-1)}
$$

Also Equation 1.2 below follows from the fact that $t_{i}^{k-1}$ is the first entry in $s\left[E_{(k-1)}\right]$ matching the time constraint.

$$
t_{i}^{k-1}<t_{j}^{k-1} \leq t, \forall j \in\{i+1 \ldots m\}
$$

From Equation 1.1 and 1.2, Equation 1.3 follows.

$$
0<t-t_{j}^{k-1} \leq t_{h i g h}^{(k-1)}, \forall j \in\{i+1 \ldots m\}
$$

This shows that every entry in $s\left[E_{(k-1)}\right]$ following $t_{i}^{k-1}$ also satisfies the interevent constraint. This follows from the relaxation of the lower-bound. Therefore it is sufficient to keep only the latest time stamp $t_{m}^{k-1}$ only in $s\left[E_{(k-1)}\right]$ since it can serve the purpose for itself and all entries above/before it, thus reducing $s\left[E_{(k-1)}\right]$ to a single time stamp rather than a list (as in Algorithm 1).

Combined Algorithm: Two-Pass Elimination. Now, we can return to the original mining problem (with both upper and lower bounds). By combining Algorithm PreElim with our hybrid algorithm, we can develop a two-pass elimination approach that can deal with the cases on which the hybrid algorithm cannot be executed. The two-pass elimination algorithm is as follows: 


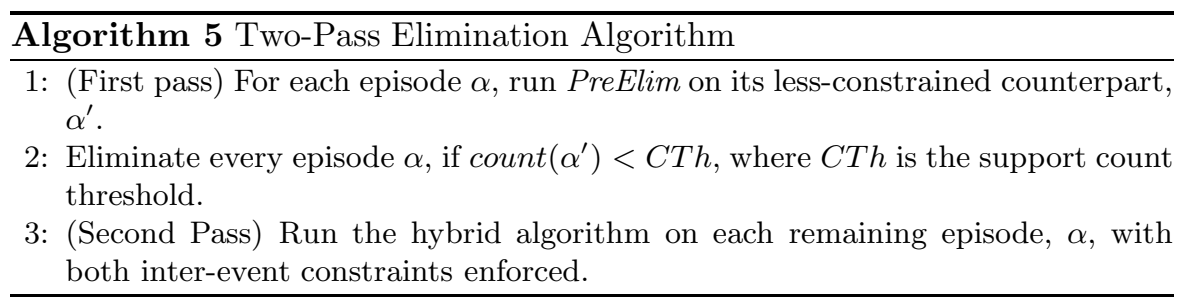

The two-pass elimination algorithm yields the correct solution for Problem 1. Although the set of episodes mined under the less constrained version are not a superset of those mined under the original problem definition, we can show the following result:

Theorem 1. count $\left(\alpha^{\prime}\right) \geq \operatorname{count}(\alpha)$, i.e., the count obtained from Algorithm PreElim is an upper-bound on the count obtained from the hybrid algorithm.

Proof. Let $h$ be an occurrence of $\alpha$. Note that $h$ is a map from event types in $\alpha$ to events in the data sequence $S$. Let the time stamps for each event type in $h$ be $\left\{t^{(1)} \ldots t^{(k)}\right\}$. Since $h$ is an occurrence of $\alpha$, it follows that

$$
t_{\text {low }}^{i}<t^{(i)}-t^{(i-1)} \leq t_{\text {high }}^{i}, \forall i \in\{1 \ldots k-1\}
$$

Note that $t_{\text {low }}^{i}>0$. The inequality in Equation 1.4 still holds after we replace $t_{\text {low }}^{i}$ with 0 to get Eqn.1.5.

$$
0<t^{(i)}-t^{(i-1)} \leq t_{\text {high }}^{i}, \forall i \in\{1 \ldots k-1\}
$$

The above corresponds to the relaxed inter-event constraint in $\alpha^{\prime}$. Therefore every occurrence of $\alpha$ is also an occurrence of $\alpha^{\prime}$ but the opposite may not be true. Hence we have that $\operatorname{count}\left(\alpha^{\prime}\right) \geq \operatorname{count}(\alpha)$.

In our two-pass elimination approach, algorithm PreElim is less complex and runs faster than the hybrid algorithm because it reduces the time complexity of the inter-event constraint check from $O\left(\left|s\left[E_{(k-1)}\right]\right|\right)$ to $O(1)$. Therefore, the performance of the two-pass elimination algorithm is significantly better than the hybrid algorithm when the number of episodes is very large and the number of episodes culled in the first pass is also large, as shown by our experimental results described next.

\subsection{Experimental Results}

\subsubsection{Datasets and Testbed}

Our datasets are drawn from both mathematical models of spiking neurons as well as real datasets gathered by Wagenar et al. [8] in their analysis of 
cortical cultures. Both these sources of data are described in detail in [6]. The mathematical model involves 26 neurons (event types) whose activity is modeled via inhomogeneous Poisson processes. Each neuron has a basal firing rate of $20 \mathrm{~Hz}$ and two causal chains of connections - one short and one longare embedded in the data. This dataset (Sym26) involves 60 seconds with 50,000 events. The real datasets (2-1-33, 2-1-34, 2-1-35) observe dissociated cultures on days 33,34 , and 35 from over five weeks of development. The original goal of this study was to characterize bursty behavior of neurons during development.

We evaluated the performance of our GPU algorithms on a machine equipped with Intel Core 2 Quad $2.33 \mathrm{GHz}$ and $4 \mathrm{~GB}$ system memory. We used a NVIDIA GTX280 GPU, which has 240 processor cores with $1.3 \mathrm{GHz}$ clock for each core, and $1 \mathrm{~GB}$ of device memory.

\subsubsection{Performance of the One Thread per Occurrence}

The best GPU implementation is compared to the CPU by counting a single episode. This is the case where the GPU was weakest in previous attempts, due to the lack of parallelization when the episodes are few. In terms of the

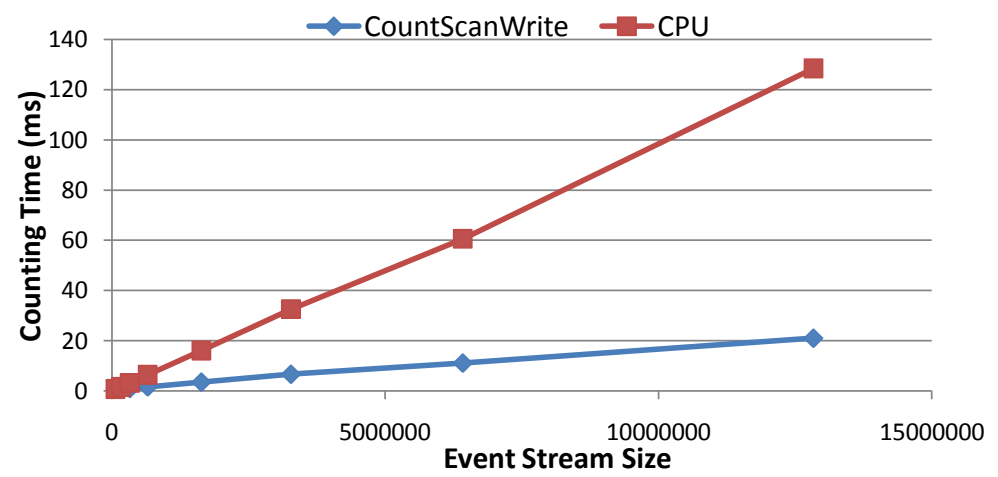

Fig. 1.6. Performance comparison of the CPU and best GPU implementation, counting a single episode in Datasets 1 through 8.

performance of our best GPU method, we achieve a $6 \mathrm{x}$ speedup over the $\mathrm{CPU}$ implementation on the largest dataset, as shown in Figure 1.6.

Figure 1.7 contains the timing information of three compaction methods of our redesigned GPU algorithm with varying episode length. Compaction using CUDPP is the slowest of the GPU implementations, due to its method of compaction. It requires each data element to be either in or out of the final compaction and does not allow for compaction of groups of elements. For small episode lengths, the CountScanWrite approach is best because sorting can be completely avoided. However, with longer episode lengths, compaction using lock-based operators shows the best performance. This method of compaction 


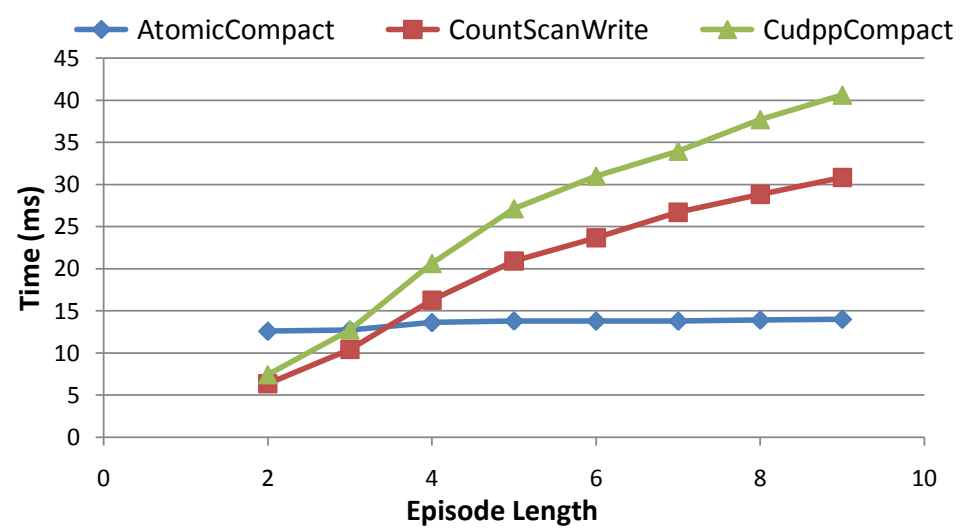

Fig. 1.7. Performance of algorithms with varying episode length in Dataset 1.

avoids the need to perform a scan and a write at each iteration, at the cost of sorting the elements at the end. The execution time of the AtomicCompact is nearly unaffected by episode length, which seems counterintuitive because each level requires a kernel launch. However, each iteration also decreases the total number of episodes to sort and schedule at the end of the algorithm. Therefore, the cost of extra kernel invocations is offset by the final number of potential episodes to sort and schedule.

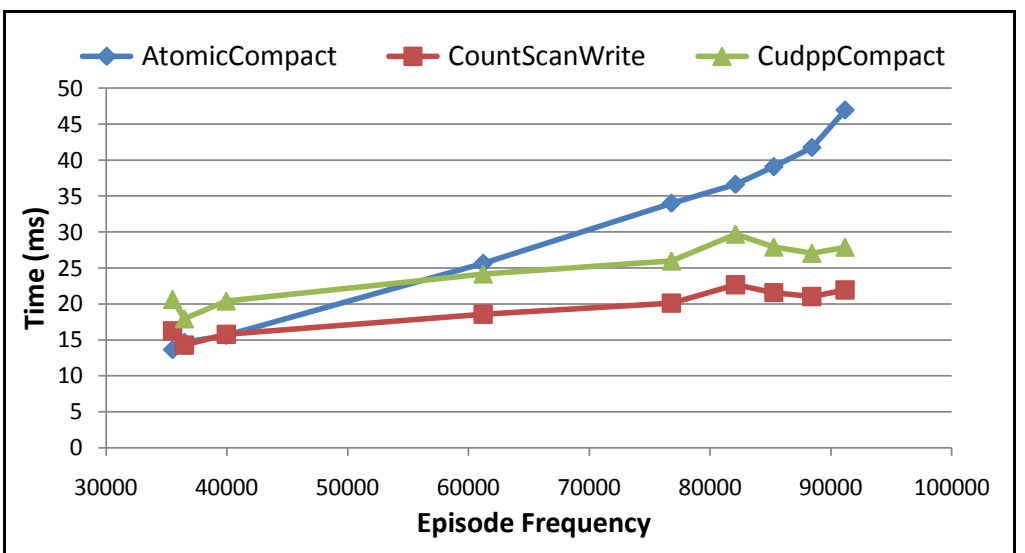

Fig. 1.8. Performance of algorithms with varying episode frequency in Dataset 1.

We find that counting time is related to episode frequency as shown in Figure 1.8. There is a linear trend, with episodes of higher frequency require more counting time. The lock-free compaction methods follow an expected trend of slowly increasing running time because there are more potential episodes to track. The method that exhibits an odd trend is the lock-based compaction, AtomicCompact. As the frequency of the episode increases, there are more po- 
tential episodes to sort and schedule. The running time of the method becomes dominated by the sorting time as the episode frequency increases.

Another feature of Figure 1.8 that requires explanation is the bump where the episode frequency is slightly greater than 80,000 . This is because it is not the final non-overlapped count that affects the running time, it is the total number of overlapped episodes found before the scheduling algorithm is applied to remove overlaps. The $\mathrm{x}$-axis is displaying non-overlapped episode frequency, where the run-time is actually affected more by the overlapped episode frequency.

We used the CUDA Visual Profiler on the other GPU methods. They had similar profiler results as the CountScanWrite method. The reason is that the only bad behavior exhibited by the method is divergent branching, which comes from the tracking step. This tracking step is common to all of the GPU methods of the redesigned algorithm.

\subsubsection{Performance of the Two-Pass Elimination Algorithm}

The performance of the hybrid algorithm suffers from the requirement of large shared memory and large register file, especially when the episode size is big. So, we introduce algorithm PreElim that can eliminate most of the nonsupported episodes and requires much less shared memory and register file, then the complex hybrid algorithm can be executed on much fewer number of episodes, resulting in performance gains. The amount of elimination that PreElim conducts can greatly affect the execution time at different episode sizes. In Figure 9(a), the PreElim algorithm eliminates over 99.9\% (43634 out of 43656) of the episodes of size four. The end result is a speedup of $3.6 \mathrm{X}$ over the hybrid algorithm for this episode size and an overall speedup for this support threshold of 2.53X. Speedups for three different datasets at different support thresholds are shown in Figure 9(b) where in every case, the twopass elimination algorithm outperforms the hybrid algorithm with speedups ranging from $1.2 \mathrm{X}$ to $2.8 \mathrm{X}$.

We also use CUDA Visual Profiler to analyze the execution of the hybrid algorithm and PreElim algorithm to give a quantitative measurement of how PreElim outperforms the hybrid algorithm on the GPU. We have analyzed various GPU performance factors, such as GPU occupancy, coalesced global memory access, shared memory bank conflict, divergent branching, and local memory loads and stores. We find the last two factors are primarily attributed to the performance difference between the hybrid algorithm and PreElim, which are shown in Figure 1.10. The hybrid algorithm requires 17 registers and 80 bytes of local memory for each counting thread, while PreElim algorithm only requires 13 registers and no local memory. Since local memory is used as supplement for registers and mapped onto global memory space, it is accessed very frequently and has the same high memory latency as global memory. In Figure 1.10 (a), the total amount of local memory access of both two-pass elimination algorithm and the hybrid algorithm comes from the 


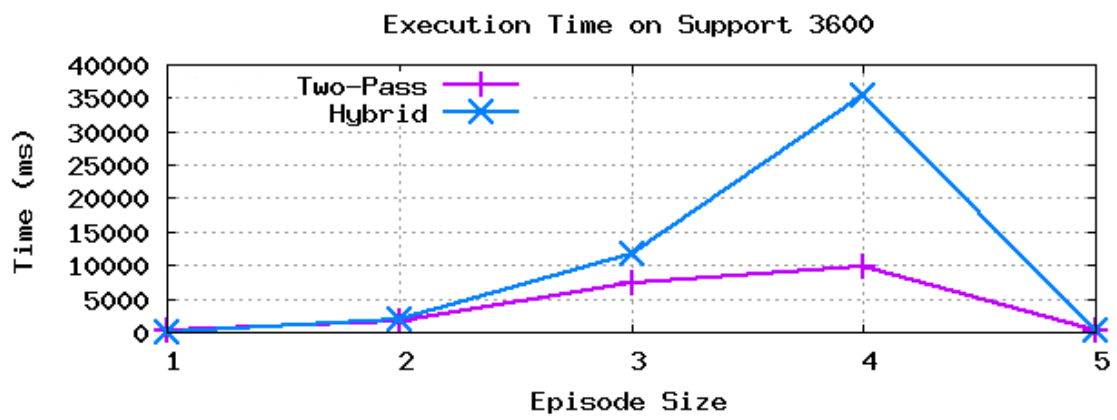

(a) Execution time of Two-Pass Elimination and Hybrid algorithms for Support $=3600$ on Dataset 2-1-35 at different episode sizes.

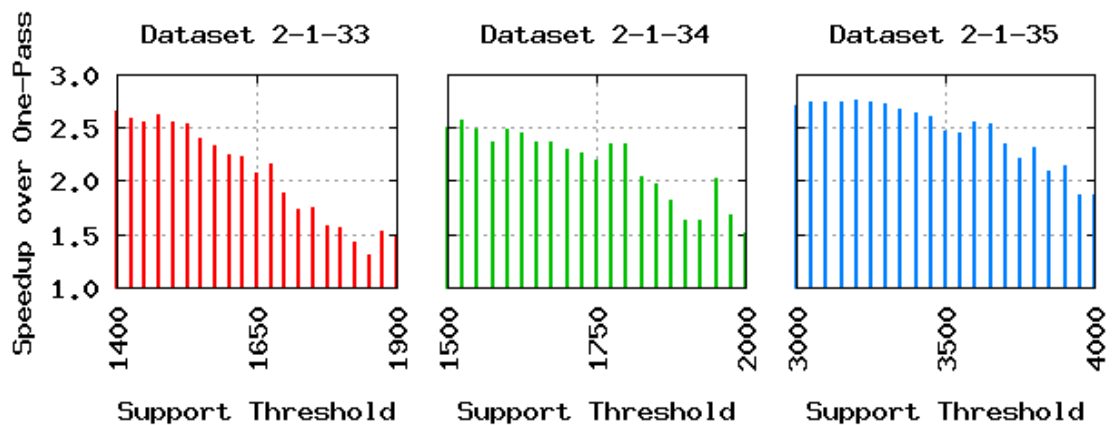

(b) Speedup of Two-Pass Elimination over Hybrid Algorithm for multiple support thresholds on multiple datasets.

Fig. 1.9. Execution time and speedup comparison of the Hybrid algorithm versus Two-Pass Elimination algorithm.

hybrid algorithm. Since the PreElim algorithm eliminates most of the nonsupported episodes and requires no local memory access, the local memory access of two-pass approach is much less than one-pass approach when the size of episode increases. At the size of 4, the PreElim algorithm eliminates all episode candidates, thus there is no execution for the hybrid algorithm and no local memory access, resulting in a large performance gain for two-pass elimination algorithm over the hybrid algorithm. As shown in Figure 1.10(b), the amount of divergent branching also affects the GPU performance difference between the two-pass elimination algorithm and the hybrid algorithm.

\subsection{Discussion}

We have presented a powerful and non-trivial framework for conducting frequent episode mining on GPUs and shown its capabilities for mining neuronal 


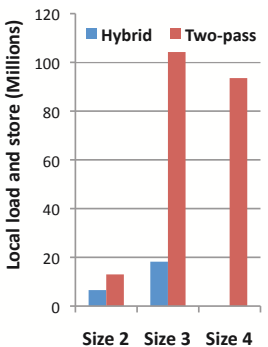

(a)

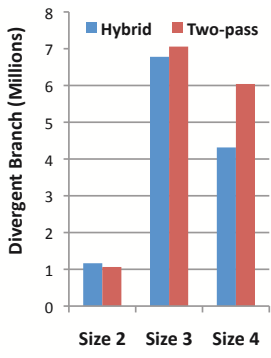

(b)

Fig. 1.10. Comparison between the hybrid algorithm and two-pass elimination algorithm for support threshold 1650 on dataset 2-1-33. (a) Total number of loads and stores of local memory. (b) Total number of divergent branches.

circuits in spike train datasets. For the first time, neuroscientists can enjoy the benefits of data mining algorithms without needing access to costly and specialized clusters of workstations. Our supplementary website (http://neuralcode.cs.vt.edu/gpgpu) provides auxiliary plots and videos demonstrating how we can track evolving cultures to reveal the progression of neural development in real-time.

Our future work is in four areas. First, our experiences with the neuroscience application have opened up the interesting topic of mapping finite state machine algorithms onto the GPU. A general framework to map any finite state machine algorithm for counting will be extremely powerful not just for neuroscience but for many other areas such as (massive) sequence analysis in bioinformatics and linguistics. Second, the development of the hybrid algorithm highlights the importance of developing new programming abstractions specifically geared toward data mining on GPUs. Third, we found that the two-pass approach performs significantly better than running the complex counting algorithm over the entire input. The first pass generates an upper bound that helps reduce the input size for the complex second pass, speeding up the entire process. We seek to develop tighter bounds that incorporate more domain-specific information about neuronal firing rates and connectivities. Finally, we wish to integrate more aspects of the application context into our algorithmic pipeline, such as candidate generation, streaming analysis, and rapid "fast-forward" and "slow-play" facilities for visualizing the development of neuronal circuits.

\section{References}

1. S. Adee. Mastering the Brain-Computer Interface. IEEE Spectrum, Apr 2008.

2. M. Harris, S. Sengupta, and J. D. Owens. GPU Gems 3, chapter Parallel prefix sum (scan) with CUDA. Addison Wesley, 2007. 
3. P. Hingston. Using finite state automata for sequence mining. Australian Computer Science Communications, Vol. 24(1):105-110, Jan-Feb 2002.

4. K. E. Iverson. A Programming Language. Wiley, New York, 1962.

5. S. Laxman, P. Sastry, and K. Unnikrishnan. A fast algorithm for finding frequent episodes in event streams. In Proc. KDD'0\%, pages 410-419, 2007.

6. D. Patnaik, P. Sastry, and K. Unnikrishnan. Inferring Neuronal Network Connectivity from Spike Data: A Temporal Data Mining Approach. Scientific Programming, 16(1):49-77, 2008.

7. S. Sengupta, M. Harris, Y. Zhang, and J. D. Owens. Scan primitives for gpu computing. In Graphics Hardware 200\%, pages 97-106. ACM, Aug. 2007.

8. D. A. Wagenaar, J. Pine, and S. M. Potter. An extremely rich repertoire of bursting patterns during the development of cortical cultures. BMC Neuroscience, 2006. 